\title{
Spatial cuing in a stereoscopic display: Evidence for a "depth-aware" attentional focus
}

\author{
PAUL ATCHLEY and ARTHUR F. KRAMER \\ University of Illinois at Urbana-Champaign, Urbana, Ilinois \\ GEORGE J. ANDERSEN \\ University of Califormia, Riverside, Califormia \\ and \\ JAN THEEUWES \\ TNO Human Factors Research Institute, Soesterberg, The Netherlands
}

\begin{abstract}
Two experiments were conducted to explore whether attentional selection occurs in depth, or whether attentional focus is "depth blind," as suggested by Ghiradelli and Folk (1996). In Experiment 1 , observers viewed stereoscopic displays in which one of four spatial locations was cued. Two of the locations were at a near-depth location and two were at a far-depth location, and a single target was presented along with three distractors. The results indicated a larger cost in reaction time for switching attention in $x, y$ and depth than in $x, y$ alone, supporting a "depth-aware" attentional spotlight. In Experiment 2, no distractors were present, similar to the displays used by Ghiradelli and Folk. In this experiment, no effect for switching attention in depth was found, indicating that the selectivity of attention in depth depends on the perceptual load imposed on observers by the tasks and displays.
\end{abstract}

The degree to which visual information is processed largely depends on the amount of attention allocated to that information. A number of metaphors have been used to describe the spatial allocation of visual attention. One of the earliest metaphors for attention was a spotlight (Broadbent, 1982; Posner, Snyder, \& Davidson, 1980; Shulman, Remington, \& McLean, 1979). The spotlight view depicts attention as an area within which all information is processed and outside of which information is largely ignored. Other studies have found that attention can operate from a narrow to a wide area of the visual field (Bashinski \& Bacharach, 1980; B. A. Eriksen \& C. W. Eriksen, 1974; Hoffman \& Nelson, 1981; Kramer \& Jacobson, 1991; LaBerge, 1983; LaBerge \& Brown, $1986)$, leading to the suggestion that attention is like a zoom lens that can be narrowly or widely spatially focused depending on the task demands (C.W. Eriksen \& St. James, 1986; C.W. Eriksen \& Yeh, 1985), or that attention functions like a gradient with maximal processing efficiency at the center and declining efficiency at more peripheral locations (Downing \& Pinker, 1985; Hughes \& Zimba, 1985; LaBerge \& Brown, 1989).

This research was supported by a cooperative research agreement with the Army Research Laboratory (DAAL0196-2-0003). We would like to thank James Cutting, Charles Folk, and Yehoshua Tsal for their helpful comments on a previous draft of this manuscript. Correspondence should be addressed to P. Atchley, Beckman Institute, University of Illinois, Urbana-Champaign, 405 W. Mathews Ave., Urbana, IL 61801 (e-mail: atchley@faroe.vp.uiuc.edu).
While the allocation of attention in two dimensions (2-D) has been well researched, only a limited number of studies have examined visual spatial attention in 3-D space. There are two general possibilities for the nature of attention in 3-D space. First, it is possible that attention may operate in 3-D much the same way it does in 2-D. Attention in 3-D space may function like a spotlight, a zoom lens, or a gradient with an extent in depth as well as in the horizontal and the vertical dimensions. If this is correct, then attending to one location in 3-D space reduces the processing of objects at other locations in depth, since those objects would be farther from the center of attention. However, a second possibility is that attention is deployed differently in depth than it is in 2-D space. For example, attention might be depth blind (Ghiradelli \& Folk, 1996) or only selectively deployed in depth when objects are present in the visual field (Hoffman \& Mueller, 1994; i.e., unlike in 2-D space, in which attention can be selectively focused even in an empty field). A number of studies suggest that attention has a 3-D spatial representation. In a study by Downing and Pinker (1985), observers viewed two rows of lights with four lights in each row. The rows of lights were arranged in depth. The observers fixated centrally and were cued to the visual location of the target. Reaction time was slower for targets that were at a different-depth plane from the cued location. This effect increased with increasing retinal eccentricity. Similar results with real 3-D scenes have also been found by Gawryszewski, Riggio, Rizzolatti, and Umiltà (1987). Both studies also found a 


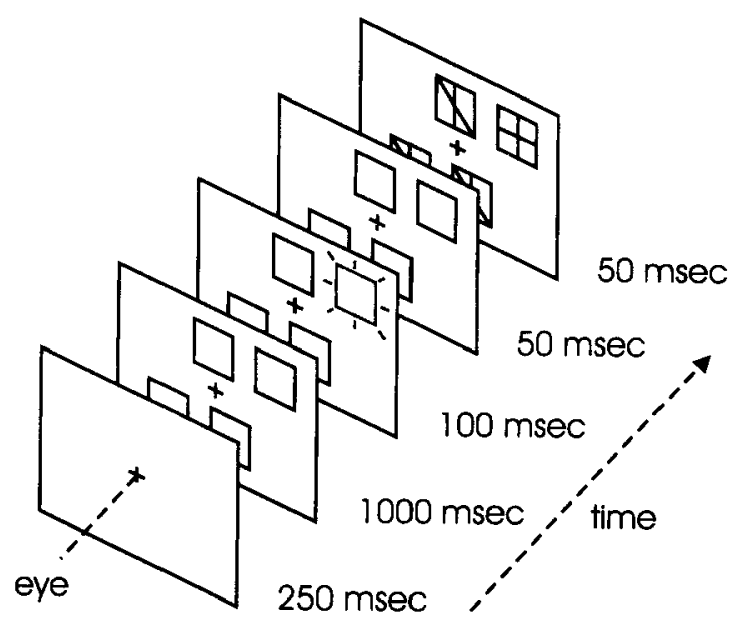

Figure 1. A schematic illustration of the displays presented in Experiment 1. The same displays were presented in Experiment 2 except that only a single target stimulus (rather than a target and three distractors) was presented.

faster reallocation of attention when switching from far to near than from near to far depth locations, suggesting that attention has a viewer-centered spatial representation. However, there are a number of potential problems with using real 3-D scenes to study spatial attention. First, placement of the lights at different distances from the observer creates the possibility of intensity and size differences in each row of lights. In such cases, selection could occur on the basis of differences in these object attributes rather than on the basis of depth per se. Second, differences in accommodation and convergence also occur when viewing real objects at different distances.

Two studies of focused attention in 3-D space have been conducted using computer displays that avoid many of the problems encountered using real 3-D scenes. In a study by Andersen (1990), observers viewed random-dot stereograms of centrally located horizontal or vertical bars with distractor bars placed nearby in $x, y$ space and at various depths relative to the target bar. Display durations were less than $120 \mathrm{msec}$ to avoid vergence eye shifts. Observer performance declined the closer the distractors were to the target in depth (see also Andersen \& Kramer, 1993). These studies suggest a gradient-like distribution of attention in depth, with maximal processing occurring at the focus and declining efficiency at more peripheral locations. The combined results of studies with real depth (Downing \& Pinker, 1985; Gawryszewski et al., 1987) and simulated depth (Andersen, 1990; Andersen \& Kramer, 1993; He \& Nakayama, 1995) suggest that attention has a spatial extent in depth. Further, most of the available evidence indicates that attention has a viewercentered representation: The cost of switching attention is higher when switching from near to far depth than from far to near depth, and the effect of distractors located near to a target in depth is greater for distractors located closer to the observer in depth than for those located far- ther from the observer. Although the studies discussed above make a strong case for a depth-aware attentional focus, there have also been a number of recent studies suggesting that attention can be depth blind (Ghiradelli \& Folk, 1996; Iavecchia \& Folk, 1995; Zimba \& Tellinghuisen, 1990). In the Ghiradelli and Folk study, for example, when observers were cued to a location in depth and the target appeared at a different location in depth but at the same $x, y$ location, no cost for switching attention was found. One potential explanation of these results is that the tasks used in these studies do not require the reallocation of attention across depth. In the Ghiradelli and Folk study, the foveal presentation of the stimuli may not have required observers to allocate attention to a particular depth location since the targets (an "=" symbol and an $x$ ) were easily distinguishable and were presented without any accompanying distractors. That is, the perceptual load may not have been sufficiently high to warrant the precise focus of attention at a specific location in depth. This hypothesis is consistent with previous reports, in 2-D attention studies, of an inverse relationship between the difficulty of perceptual discriminations and the breadth of the attentional focus. A more precise focus of attention, indexed by a decrease in distractor interference in the flanker paradigm (B. A. Eriksen \& C. W. Eriksen, 1974), occurs with increases in the number of distractors surrounding a target (Miller, 1991; Yantis \& Johnston, 1990) as well as with more difficult target discriminations (Lavie, 1995). Indeed, such results have led Lavie and Tsal (1994) to suggest that "early selection is possible only when the perceptual load of processing the relevant information is sufficiently high to approach or exceed the upper limit of the total available resources. If this condition is not met, spare capacity will be unintentionally allocated for the irrelevant processing, thus preventing early selection" (p. 194). We speculate that Ghiradelli and Folk's (1996; see also Iavecchia \& Folk, 1995; Zimba \& Tellinghuisen, 1990) failure to find performance costs when a target occurred in an uncued location in depth might be attributable to the easily distinguishable target stimuli coupled with the lack of distractors. Thus, the task may not have required a precise focus of attention, thereby resulting in the allocation of attention to both the cued and uncued depth locations.

We tested this perceptual load hypothesis in the present studies by having observers discriminate between two relatively similar targets, an $\mathrm{x}$ and a cross, that were presented along with distractors containing features also found in the targets (e.g., a vertical line and an oblique line; see Figure 1). Observers viewed contour stereograms through a set of liquid crystal shutter glasses. Each trial consisted of a fixation point, a precue display with four empty boxes arranged in a $2 \times 2$ grid around the fixation point, a cue display in which one of the boxes briefly increased in luminance, and a target display in which the observers were required to indicate which of two targets appeared. The target appeared in one location and dis- 
tractors appeared at the other three locations. The boxes were arranged in depth so that two of the boxes were farther in depth relative to the fixation point and two of the boxes were closer in depth. On some of the trials, an invalid cue (a cue that indicated the incorrect $x, y$ location of where the target would appear) was presented. On some of the invalid trials, the target appeared at the samedepth plane (invalid-same depth) and on others it appeared at a different-depth plane (invalid-different depth) from the cue.

If the relatively high perceptual load engendered by the need to make a difficult discrimination between targets presented along with physically similar distractors is sufficient to encourage observers to precisely focus their attention in 3-D space, then the cost for switching attention (an increase in reaction time [RT] and/or a decrease in accuracy) should be greater in the differentdepth condition than in the same-depth condition. On the other hand, if attention is "depth blind," as suggested by Ghiradelli and Folk (1996), the cost for switching attention should be no greater for the same- or different-depth uncued locations.

\section{EXPERIMENT 1}

\section{Method}

\section{Observers}

A total of 14 observers recruited at the University of Illinois at Urbana-Champaign participated in two 30 -min sessions across 2 days. The observers were paid $\$ 5$ per hour for their participation. All observers were screened for adequate stereopsis with a customized screening procedure (described below) and had normal or correctedto-normal vision (20/30 or better).

\section{Apparatus}

The stereographic displays were presented on a Magnavox 21 -in. monitor. Binocular fusion was achieved by presenting separate left and right eye images with a phase haploscope, using a set of liquid crystal shutter glasses (Stereographics Corp., Mountain View, CA). The frame rate was $120 \mathrm{~Hz}(60 \mathrm{~Hz}$ per eye). The displays were presented with a Pentium-based computer system (running at $200 \mathrm{MHz}$ ) using a Matrox Millenium graphics card. The displays were viewed in a darkened room at a distance of $750 \mathrm{~mm}$. A chinrest was used to stabilize the observer's head.

\section{Stimuli}

Contour stereograms were used in both the stereo screening test and the experimental trials. Four types of displays were used: a fusion display, a precue display, a cue display, and a response display. The fixation point consisted of a red cross. The vertical and horizontal bars that defined the cross subtended $0.20^{\circ} \times 0.57^{\circ}$ and $0.18^{\circ} \times 0.57^{\circ}$, respectively. The fixation point was presented without disparity and appeared at the depth of the monitor. The precue display consisted of four empty boxes arranged in a $2 \times 2$ grid around the fixation display. Each box subtended $3.92^{\circ} \times 3.92^{\circ}$. The outline of the box was $0.20^{\circ}$ thick (vertical lines) and $0.21^{\circ}$ thick (horizontal lines). The luminance of the outline was $5.7 \mathrm{~cd} / \mathrm{m}^{2}$ and the luminance of the background was $.1 \mathrm{~cd} / \mathrm{m}^{2}$. The distance from the nearest corner of the box to the center of fixation was $0.32^{\circ}$. On each trial, two of the boxes were presented at crossed disparities relative to fixation and two at uncrossed disparities relative to fixation, so that two of the boxes appeared closer to the observer in depth than the fixation point and two appeared more distant. The disparity values used were + and -25 arc min. The cue display consisted of increasing the luminance of one box to $8.8 \mathrm{~cd} / \mathrm{m}^{2}$. On neutral trials, all boxes were highlighted. The target display con- sisted of the four boxes without the fixation point. One location contained a target that was either an $\mathrm{x}$ or a cross. The $\mathrm{x}$ was formed from two diagonai lines from each of the corners of the box. The cross was made up of two lines, each $0.20^{\circ}$ thick, extending from the top center to the bottom center and the left center to the right center of the box. The lines were $0.21^{\circ}$ thick. On experimental trials, the other three locations contained distractors. The distractors consisted of a diagonal line from the top left to the bottom right and a vertical line from the top center to the bottom center, each with the same thickness as its target counterparts.

\section{Design}

Two within-observers factors were used: cue validity (valid, neutral, invalid same depth, invalid different depth) and cue location (near and far). Four depth layouts (far boxes on top, on bottom, on left, or on right) and four target locations (upper right, lower right, lower left, and upper left) were counterbalanced across all conditions. Each session consisted of 576 trials. One third of the trials were neutral. For the remaining trials, $75 \%$ were valid and $25 \%$ were invalid. On both invalid cue conditions (same or different depth), the target was in a different $x, y$ location than the cue. In the same-depth condition, the cue and target were at the same depth, whereas in the different-depth condition they were at opposite depths. The parameters for each trial were randomized within the constraints of the design. Trials on which the observer responded faster than $200 \mathrm{msec}$ or slower than $1,500 \mathrm{msec}$ were discarded and rerun at the end of the experiment.

The distance from the cue to the invalid target location was equivalent for the same- and different-depth trials. For example, consider the case in which the two boxes on the left were at the near depth and the two boxes on the right were at the far depth. Assuming that the cued location was the box on the top left, the target on the invalid location trials would have an equal probability of occurring at a near depth (lower left box) or far depth (upper right box). Diagonal locations were not used.

\section{Procedure}

Screening procedure. Observers were required to pass a custom stereo screening test designed to assess their ability to perceive depth in the experimental displays. The screening test was presented with the same apparatus and under the same conditions as in the experiment. The same fusion display/fixation point and precue displays were used as in the experimental trials. Two blocks of 32 trials per block were conducted. Each block had all possible combinations of depth layout and target locations and target types. The screening trials consisted of the presentation of a fixation display for $250 \mathrm{msec}$ followed by a precue display for $1,000 \mathrm{msec}$. The fixation point was then removed and an $x$ appeared in one of the four boxes. The target display was removed after $200 \mathrm{msec}$. The observer's task was to verbally indicate at which depth plane (near or far relative to the observer) the target appeared. A bell sounded for each incorrect answer. Observers were not required to indicate the type of target that appeared, but were told that these would be the targets that they would be looking for during the experiment. The first block was used as practice. To be included in the study, the observers were required to respond with the correct depth 10 times in a row during the second block of trials.

Experimental procedure. Observers participated in two sessions over 2 days. The first session lasted 45 min (including the custom depth screening procedure) and was used as practice. The data from this session were not included in the analysis. The second session lasted $30 \mathrm{~min}$. Observers were instructed to maintain fixation on the fixation point and to respond as quickly and as accurately as possible. The observers were encouraged to take breaks as often as they liked.

At the beginning of each trial, the fixation point was presented for $250 \mathrm{msec}$, followed by the precue display for $1,000 \mathrm{msec}$. On cue trials, following the precue display, one location was cued for $100 \mathrm{msec}$. On neutral trials, all locations were cued for $100 \mathrm{msec}$. After a $50-\mathrm{msec}$ interstimulus interval (ISI), the fixation point was removed and the target display (target and distractors) was presented for $50 \mathrm{msec}$. The time from the start of the cue display to the offset of the target display was $200 \mathrm{msec}$, which is near the lower time limit required to initiate a vergence eye shift (Rashbass \& Westheimer, 1961; Westheimer \& 


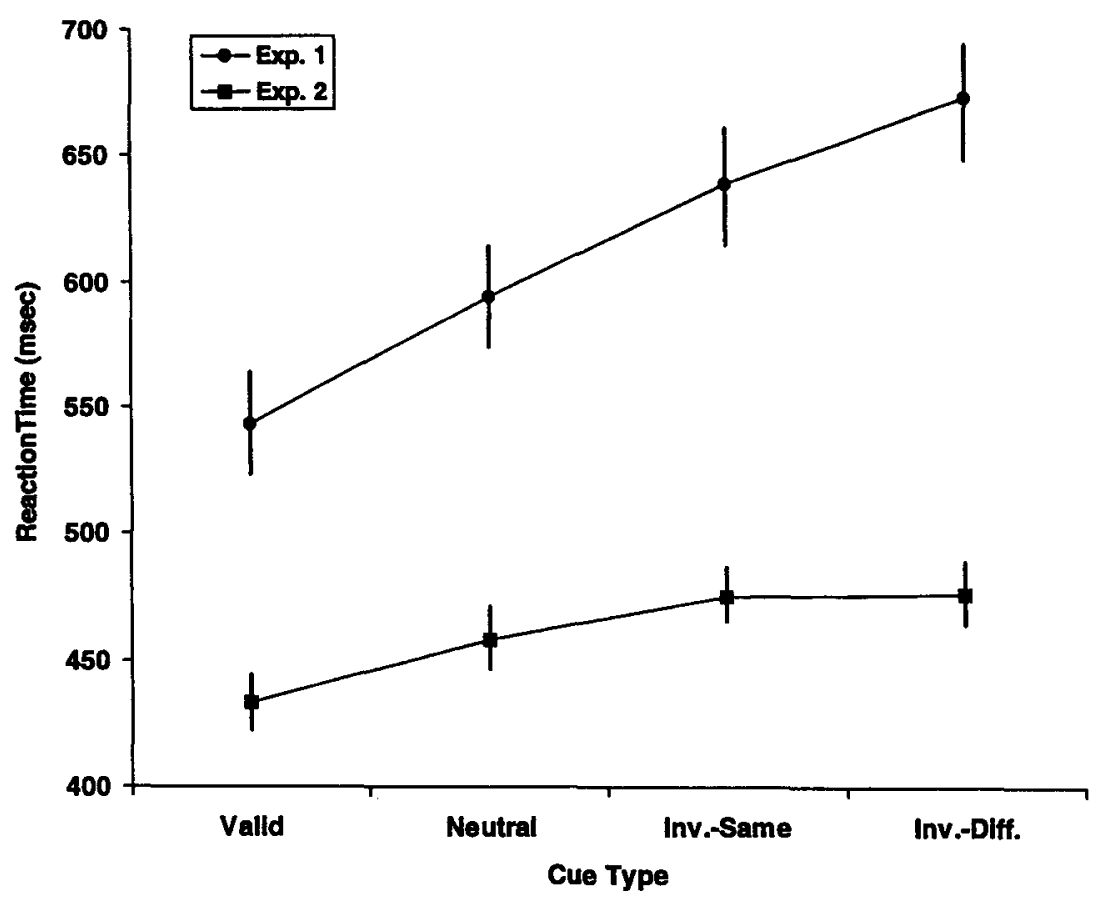

Figure 2. The mean reaction times for each of the conditions in Experiments 1 (filled circles) and 2 (filled squares) along with standard error bars.

Mitchell, 1969). Observers responded by pressing the right mouse button if they detected an $\mathrm{x}$ or the left mouse button if they detected a cross. Incorrect trials were followed by a $500-\mathrm{msec}$ tone. The interval between trials was $1,000 \mathrm{msec}$.

\section{Results and Discussion}

Fourteen observers were run to obtain 10 who passed the screening procedure. Both RT for correct responses and mean accuracy rates from the 10 observers were analyzed with a 4 (cue type) $\times 2$ (cue location) analysis of variance (ANOVA). Mean RT for each cue type is shown in Figure 2. The main goal of this study was to determine whether the "cost" for reorienting attention from an invalidly cued location to an uncued location would be larger if the uncued location occurred at a different depth than that of the cued location. That is, we were interested in whether attention selection occurs in depth as it does in $x, y$ space.

As can be seen in Figure 2, RTs increased from the valid to the neutral to the invalid same-depth and finally to the invalid different-depth condition. This observation was supported by a significant main effect for cue type $[F(3,27)=39.41, p<.001]$. Consistent with our hypothesis that attentional selection occurs in depth, at least under conditions of high perceptual load, we found that RTs were significantly slower for the invalid different-depth $(672 \mathrm{msec})$ than for the invalid same-depth $(639 \mathrm{msec})$ trials. ${ }^{1}$ Also consistent with many previous studies of attention in 2-D space (Posner et al., 1980; Shulman et al., 1979), mean RTs were also significantly faster on the valid trials $(542 \mathrm{msec})$ than on the neutral cue trials $(593 \mathrm{msec})$.
Overall, accuracies were very high $(>94 \%$ correct in each of the experimental conditions). However, we did obtain a significant interaction between cue type and depth location $[F(3,27)=9.15, p<.001]$. For the invalid different-depth trials, performance was significantly more accurate when the target was at the near-depth location (mean error $=2.2 \%$ ) than at the far-depth location (mean error $=5.0 \%$ ). This pattern of errors is consistent with findings from other experiments (Downing \& Pinker, 1985; Gawryszewski et al., 1987) in which attention was more quickly reoriented from far to near locations than from near to far locations. Furthermore, the present results are consistent with a viewer-centered attentional gradient in which attention is allocated from the observer to the point of fixation (or, in the present case, vergence). When attention needs to be reallocated outside of this representation, as would be the case when a target occurs at an uncued location distant from the observer, performance is poorer than when attention must be reoriented to a location between fixation and the observer.

\section{EXPERIMENT 2}

The results of our first experiment do indeed suggest, contrary to the findings reported by Ghiradelli and Folk (1996; see also Iavecchia \& Folk, 1995; Zimba \& Tellinghuisen, 1990), that attentional selection in spatial cuing tasks can indeed operate on a representation that includes depth. However, given our speculation that previous failures to find evidence of such selectivity can be attributed to the relatively low perceptual load imposed on subjects 
by the tasks and displays employed in those studies, we believed it important to conduct another experiment in which perceptual load was reduced (Miller, 1991; Yantis \& Johnston, 1990). We accomplished this by presenting observers with displays that were similar to those in Experiment 1 with one exception: The distractors were not presented when the target appeared. In this experiment, the target was the only item in the last frame of the display (besides the placeholders). Given the reduced perceptual load in this study, we anticipated that it would not be necessary for the observers to narrowly focus their attention at the target location, thereby reducing the performance costs when the target appears at an uncued depth. Such an effect would provide additional support for the proposal that early selection-in the present case, selection on the basis of the location of the target in 3-D space - is more likely to occur under conditions of high than low perceptual load (Lavie, 1995; Lavie \& Tsal, 1994).

\section{Method}

\section{Observers}

A total of 12 observers recruited at the University of Illinois at Urbana-Champaign participated in two 30-min sessions across 2 days. The observers were paid $\$ 5$ per hour for their participation. All observers were screened for adequate stereopsis with a customized screening procedure (previously described) and had normal or correctedto-normal vision $(20 / 30$ or better $)$

\section{Apparatus, Stimuli, Design, and Procedure}

All aspects of this experiment were similar to those of Experiment 1 with the following exception. During the target phase, the target was presented (an $\mathrm{x}$ or a cross) without distractors. The placeholders for the nontarget locations remained present.

\section{Results and Discussion}

Twelve observers were run to obtain 10 who passed the screening procedure. Both mean RT for correct responses and mean accuracy rates from the 10 observers were analyzed with a 4 (cue type) $\times 2$ (cue location) ANOVA. Mean RT for each cue type is shown in Figure 2. As can be seen in the figure, the differences among cue conditions, although statistically significant $[F(3,27)=$ $32.62, p<.001]$, were substantially less than those found in Experiment 1. The important question, however, is whether the reduction in perceptual load engendered by the removal of the distractors in the present study (and therefore similar to the displays employed in previous studies, such as those by Ghiradelli \& Folk, 1996, Iavecchia \& Folk, 1995, and Zimba \& Tellinghuisen, 1990), diminished the performance costs associated with the need to reallocate attention in depth. That this was indeed the case is suggested by the nonsignificant difference in RT between the invalid same $(475 \mathrm{msec})$ and invalid different $(476 \mathrm{msec})$ conditions $[F(1,9)=0.81]$. Thus, our results replicate the findings reported by other researchers of a failure to find performance costs when a lone target appears at an uncued location in depth. Interestingly, and also consistent with other researchers, we did obtain a significant difference between the valid
(433 msec) and neutral ( $458 \mathrm{msec}$ ) and neutral and combined $(476 \mathrm{msec})$ invalid same- and invalid differentdepth conditions. Thus, even in the absence of distractors, there were significant costs for reorienting attention to different positions within a depth plane. As in Experiment 1 , accuracies were quite high in all of the experimental conditions. The mean error rates for the valid, neutral, invalid same-depth, and invalid different-depth conditions were $3.0 \%, 3.4 \%, 3.8 \%$, and $2.7 \%$, respectively. These differences were not statistically significant.

\section{GENERAL DISCUSSION}

Two experiments were conducted to explore the representation of depth in visual spatial attention. In the first experiment, observers were cued to a location in $x, y$, and $z$ (depth) space where a target might appear. The target was surrounded by three similar distractors. When the target appeared at an $x, y$ location that was different from the cued location, the performance cost was greater when observers were also required to shift their attention to a different depth location than when they were simply required to shift their attention in $x, y$ space. This experiment clearly demonstrates that attention can be localized to a location in depth. In a second experiment, in which we used a similar paradigm minus the distractors (á la Ghiradelli \& Folk, 1996), we did not find any evidence for selective attention in depth. The results of these two experiments, when considered together, suggest that previous studies that have failed to find evidence of selective attention in depth in spatial cuing tasks (Ghiradelli \& Folk, 1996; Iavecchia \& Folk, 1995; Zimba \& Tellinghuisen, 1990) have likely done so as a result of the relatively low perceptual loads imposed on subjects in those studies. That is, in the absence of distractors that are similar in appearance to the targets and with easily distinguishable targets, narrowly focused attention is not necessary to perform the task, thereby precluding the need to reorient attention between cued and uncued locations in depth (Lavie, 1995; Lavie \& Tsal, 1994). Thus, the results of our study provide a resolution to the paradox in the literature as to why some studies have yielded evidence for selective attention in depth (Andersen \& Kramer, 1993; He \& Nakayama, 1995; Hoffman \& Mueller, 1994; Nakayama \& Silverman, 1986) whereas others have not (Ghiradelli \& Folk, 1996; Iavecchia \& Folk, 1995; Zimba \& Tellinghuisen, 1990). Observers appear to selectively attend to locations in depth whenever they must search for a target among a multitude of distractors (He \& Nakayama, 1995; Nakayama \& Silverman, 1986), perform a focused attention task in the presence of surrounding distractors (Andersen, 1990; Andersen \& Kramer, 1993), attend to objects defined with pre-masks (Hoffman \& Mueller, 1994), and, as in the present case, reorient attention, in the presence of distractors, between cued and uncued locations.

There is, however, an aspect of our results that merits some additional discussion. As can be seen in Figure 2, results obtained in our two studies differ not only in terms of the size of the invalid differentdepth effect (i.e., invalid different depth-invalid same depth), but also in terms of the main effect of RT. That is, RTs obtained in the absence of distractors (Experiment 2) were also substantially faster than those obtained when distractors were present in the display (Experiment 1 ). Thus, it might be suggested that our finding evidence for selective attention in depth is not the result of differential perceptual load in the two studies, but instead is due to the differences in mean RTs between the studies. That is, it could be that attention in depth effects might only become apparent with slow responding. Although we consider such an account unlikely, especially given that costs and benefits for spatial cuing in 2-D space are obtained with even faster RTs than those observed in our Experiment 2 (Posner et al., 1980), we addressed this issue by examining RTs in the upper quartile of the RT distribution for Experiment 2. We reasoned that if we still failed to observe differences in RTs between invalid same- and invalid different-depth conditions with these slower RTs, this alternative explanation of our results would be less likely. Indeed, we found that this was the case on 
examination of the RTs from the upper quartile of RT distributions obtained in Experiment 2. Mean RTs for the valid, neutral, invalid same-, and invalid different-depth conditions were 491, 519, 529, and $528 \mathrm{msec}$, respectively. Although there was a significant difference between the valid and invalid same-depth conditions $[t(9)=8.44, p<$ $.01]$, there was no significant difference between the invalid same- and invalid different-depth conditions $[t(9)=.22]$. Thus, there appears to be little evidence for the slowed responding hypothesis (i.e., independent of the manipulation of perceptual load).

Although our studies, and the research previously reported by others, suggests that attention operates in depth, there are a number of unanswered questions that suggest a need for additional research on the characteristics of attention in 3-D space. One question concerns the representation in which attention operates--whether it is viewer centered (Andersen \& Kramer, 1993; Downing \& Pinker, 1985; Gawryszewski et al., 1987), action centered (Tipper, Lotie, \& Baylis, 1992), or possibly object centered (Kramer, Weber \&,Watson, 1997; Vecera \& Farah, 1994). Indeed, the answer to this question may very well depend on the nature of the task as well as observers' processing strategies. Another interesting and important question is whether attention in 3-D space is allocated to particular regions of visual space (C. W. Eriksen \& Yeh, 1985; LaBerge \& Brown, 1989) or is instead allocated to specific objects that appear in the visual field (Duncan, 1984; Kramer \& Jacobson, 1991; Vecera \& Farah, 1994). A third question concerns whether other types of load manipulations-for example, conceptual rather than perceptual load, as was manipulated in our studies - is sufficient to encourage observers to selectively focus attention in depth or whether the expectancy of distractors being present rather than their actual appearance in the visual field is sufficient to encourage a narrow attentional focus in depth. Finally, it would seem important to determine why narrowly focused attention occurs in the absence of distractors in 2-D space (Posner et al., 1980) but not in depth, as found in the present study and in Ghiradelli and Folk (1996). One possibility is that reorientation of attention in depth, at least within the confines of Panum's fusion area, is more costly to the attentional system than is reorientation in 2-D space. We are currently exploring these issues in our laboratory.

\section{REFERENCES}

ANDERSEN, G. J. (1990). Focused attention in three-dimensional space. Perception \& Psychophysics, 47, 112-120.

ANDERSEN, G. J., \& KRAMER, A. F. (1993). Limits of focused attention in three-dimensional space. Perception \& Psychophysics, 53, 658 667

BASHINSKI, H. S., \& BACHARACH, V. R. (1980). Enhancement of perceptual sensitivity as the result of selectively attending to spatial locations. Perception \& Psychophysics, 28, 241-248.

BROADBENT, D. (1982). Task combination and selective intake of information. Acta Psychologica, 50, 253-290.

Downing, C., \& PINKER, S. (1985). The spatial structure of visual attention. In M. I. Posner \& O. S. M. Martin (Eds.), Attention and performance $X I$ (pp. 171-187). Hillsdale, NJ: Erlbaum.

DUNCAN, J. (1984). Selective attention and the organization of visual information. Journal of Experimental Psychology: General, 113, 501-517.

Eriksen, B. A., \& EriKsen, C. W. (1974). Effects of noise letters upon the identification of a target letter in a nonsearch task. Perception \& Psychophysics, 16, 143-149.

ERIKSEN, C. W., \& ST. JAMES, J. D. (1986). Visual attention within and around the field of focal attention: A zoom lens model. Perception \& Psychophysics, 40, 225-240.

ERIKSEN, C. W., \& YeH, Y. (1985). Allocation of attention in the visual field. Journal of Experimental Psychology: Human Perception \& Performance, 11, 583-589.

Gawryszewski, L. D. G., Riggio, L., Rizzolatti, G., \& Umiltà, C (1987). Movements of attention in three spatial dimensions and the meaning of "neutral" cues. Neuropsychologica, 25, 19-29.

Ghiradelli, T. G., \& Folk, C. L. (1996). Spatial cuing in a stereoscopic display: Evidence for a "depth-blind" attentional spotlight. Psychonomic Bulletin \& Review, 3, 81-86.
He, Z. J., \& Nakayama, K. (1995). Visual attention to surfaces in three-dimensional space. Proceedings of the National Academy of Sciences, 92, 11155-11159.

Hoffman, J. E., \& Mueller, S. (1994, November). An in depth look at visual attention. Paper presented at the annual meeting of the Psychonomic Society, St. Louis.

HofFMAn, J. E., \& Nel SON, B. (1981). Spatial selectivity in visual search. Perception \& Psychophysics, 30, 283-290.

Hughes, H., \& ZimBa, L. (1985). Spatial maps of directed visual attention. Journal of Experimental Psychology: Human Perception \& Performance, 11, 409-430.

IAVECCHIA, H. P., \& FOLK, C. L. (1995). Shifting visual attention in stereographic displays: A timecourse analysis. Human Factors, 36, 606-618.

Kramer, A. F., \& Jacobson, A. (1991). Perceptual organization and focused attention: The role of objects and proximity in visual processing. Perception \& Psychophysics, 50, 267-284.

Kramer, A. F., WEBER, T. A., \& WATSON, S. E. (1997). Object-based attentional selection-grouped arrays or spatially invariant representations? Journal of Experimental Psychology: General, 126, 3-13.

LABERGE, D. (1983). Spatial extent of attention to letters in words. Journal of Experimental Psychology: Human Perception \& Performance, 9, 371-379.

LABERGE, D., \& BROWN, V. (1986). Variations in the size of the visual field in which targets are presented: An attentional range effect. Perception \& Psychophysics, 40, 188-200.

LABERGE, D., \& BROWN, V. (1989). Theory of attentional operations in shape identification. Psychological Review, 96, 101-124.

Lavie, N. (1995). Perceptual load as a necessary condition for selective attention. Journal of Experimental Psychology: Human Perception \& Performance, 21, 451-468.

Lavie, N., \& Tsal, Y. (1994). Perceptual load as a major determinant of the locus of selection in visual attention. Perception \& Psychophysics, 56, 183-197.

MilLer, J. (1991). The flanker compatibility effect as a function of visual angle, attentional focus, visual transients, and perceptual load: A search for boundary conditions. Perception \& Psychophysics, 49, 270-288.

Nakayama, K., \& Silverman, G. H. (1986). Serial and parallel processing of visual feature conjunctions. Nature, 320, 264-265.

Posner, M. I., SNYder, C. R., \& DAvidson, B. J. (1980). Attention and the detection of signals. Journal of Experimental Psychology: General, 109, 160-174.

Rashbass, C., \& Westheimer, G. (1961). Independence of conjugate and disjunctive eye movements. Journal of Physiology, 159, 361-364.

Shulman, G., Remington, R., \& McLean, J. (1979). Moving attention through visual space. Journal of Experimental Psychology: Human Perception \& Performance, 5, 522-526.

TIPPER, S. P., LOTIE, C., \& BAYLIS, G. C. (1992). Selective reaching: Evidence for action centered attention. Journal of Experimental Psychology: Human Perception \& Performance, 18, 891-905.

VECERA, S., \& FARAH, M. (1994). Does attention select objects or locations? Journal of Experimental Psychology: General, 123, 146-160.

Westheimer, G., \& Mitchell, D. E. (1969). The sensory stimulus for disjunctive eye movements. Vision Research, 9, 392-405.

YANTIS, S., \& JohNSTON, J. (1990). On the locus of visual selection: Evidence from focused attention tasks. Journal of Experimental Psychology: Human Perception \& Performance, 16, 135-149.

Zimba, L., \& Tellinghuisen, D. J. (1990, November). The covert orienting of attention to stereoscopic targets. Poster presented at the annual meeting of the Psychonomic Society, New Orleans.

\section{NOTE}

1. All of the post hoc comparisons performed in Experiments 1 and 2 were performed with the Duncan multiple range test and were significant at $p<.05$.

(Manuscript received February 25, 1997; revision accepted for publication June 9,1997 .) 